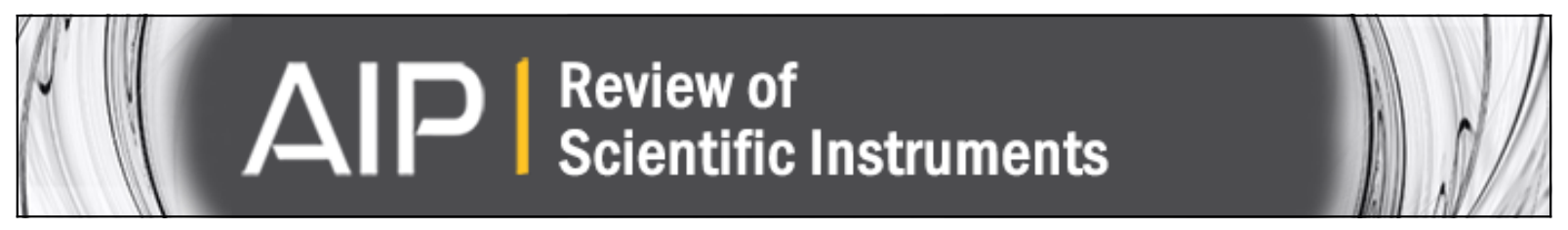

\title{
An acoustic position sensor
}

Shuxiang Dong, Feiming Bai, Jie-Fang Li, and Dwight Viehland

Citation: Review of Scientific Instruments 74, 4863 (2003); doi: 10.1063/1.1614850

View online: $\mathrm{http}: / / \mathrm{dx}$.doi.org/10.1063/1.1614850

View Table of Contents: http://scitation.aip.org/content/aip/journal/rsi/74/11?ver=pdfcov

Published by the AIP Publishing

\section{AlP Re-register for Table of Content Alerts}




\title{
An acoustic position sensor
}

\author{
Shuxiang Dong, ${ }^{\text {a) }}$ Feiming Bai, Jie-Fang Li, and Dwight Viehland \\ Materials Science and Engineering, Virginia Tech, Blacksburg, Virginia 24061
}

(Received 6 March 2003; accepted 30 July 2003)

This article presents an acoustic method-the resonance acoustic field sensor or piezoelectric-sound-resonance cavity (PSRC) - for object position detection. This method utilizes the change of acoustic radiation impedance as a sensing mechanism. The PSRC both generates and detects a resonance acoustic field, along both the axial and transverse directions. We have discovered that an inserted object or an object motion in the sound radiation field results in changes in both the voltage and phase of the PSRC. Results have shown that a minimum object displacement of $<10 \mu \mathrm{m}$ can be detected in the axial direction (and $<100 \mu \mathrm{m}$ in the transverse) by this method.

(C) 2003 American Institute of Physics. [DOI: 10.1063/1.1614850]

\section{INTRODUCTION}

Recently, there has been considerable research activity in the development of position and object detection sensors in air for industrial robotics, vehicle guidance systems, and in situ process monitoring technologies. ${ }^{1-17}$ Current noncontact position/object sensor technologies include: (i) fine position inductive types that operate at short ranges, such as eddy current, magnetoresistive, and Hall effect sensors; (ii) optical time of flight and optical coherence position sensors such as laser interferometery, optical triangulation, and optical fiber methods; $;{ }^{12-14}$ and (iii) acoustic or ultrasonic sensors.

Current noncontact acoustic or ultrasonic position/object detections are based on time-of-flight or time-delay estimation using (i) pulse mode $e^{2-7,9,11}$ and (ii) continuous wave (cw) mode ${ }^{15-18}$ In pulse mode, acoustic sonar sensors or arrays actively transmit and receive reflected acoustic waves (echoes) from objects. This technology measures the transit time of echoes, and estimates the object distance by assuming that the sound velocity in the propagation medium (air or water) is known. Standard room temperature ultrasonic technology in air can resolve a position within $\sim 0.02 \mathrm{~mm}$ over ranges exceeding $100 \mathrm{~mm}^{8}$. To obtain a higher position resolution, or to monitor a fast-changing object, normally a higher frequency sharp pulse is required. In the cw mode, the time-delay information is obtained by measuring the phase shift with a pair of transmitter and receiver ${ }^{15,18}$ or a multifrequency excitation and detection sensor system. ${ }^{16,17}$ Though other methods (i.e., inductive and optical) are available, the acoustic echo method remains important for position/object sensing in many circumstances, where other methods have proven difficult or cost ineffective. ${ }^{8,10}$

Here, we report the development of a high-resolution piezoelectric-sound-resonance-cavity (PSRC) sensor for generation of resonance acoustic fields, and subsequent detection of resonance state and impedance changes of the PSRC caused by objects or their motion. This method is significantly different from conventional echo methods. It offers

${ }^{\text {a)} E l e c t r o n i c ~ m a i l: ~ s d o n g @ v t . e d u ~}$ higher resolution and the ability to detect both object insertion and object motion.

\section{OPERATIONAL PRINCIPLE}

Figure 1 illustrates the concept of a PSRC sensor. A disk-type piezoelectric thin layer/metal laminate that has a center-opening is placed at one end of a small cylindrical cavity. A capacitance type minisound sensor is then placed at the other end of the cavity. The piezoelectric film is used to produce a bending vibration under a small applied alternating current (ac) voltage signal, forcing the acoustic medium (air or water) in the cavity to vibrate in a sound resonance state. A resonance acoustic field forms in the cavity, which radiates out from a center-opening in the piezoelectric/metal laminate disk, along the axial or $\hat{z}$ direction of the cylindrical cavity. The minisound sensor at the opposite end of the cavity monitors pressure oscillations inside the cavity. The minisound sensor produces a voltage signal $\left(V_{s}\right)$ and phase $(\theta)$ which are proportional to the acoustic intensity $(I)$.

The PSRC sensing mechanism is based on the fact that when an object is placed near an acoustic wave radiation field, reflections are produced from the object. This disturbs the initial acoustic radiation field. The reflected wave also causes a change in the acoustic radiation impedance, including the radiation resistance $\Delta R_{r}$ and attachment radiation mass $\Delta M_{r}$. For some special positions $\left[N\left(\lambda_{\text {air }} / 2\right), N\right.$ $=1,2,3 \ldots]$, the reflected waves interfere with incident radiation waves, generating new standing waves. Consequently, the acoustic resonance state (frequency and phase) of the cavity is altered. By using an acoustic sensor to monitor changes in the sound resonance field of the cavity, the static and dynamic positions of an object can be detected.

Suppose that the piezoelectric disc vibrates under an ac voltage $V_{E}$. This forces the air in the cavity to contract and expand in a cyclical mode, as shown in Fig. 2. The air vibrations in the cavity will then force the air column in the center-opening to vibrate too, due to a pressure differential inside the cavity. The vibration distribution $w(r)$ of the piezoelectric disk along its radial direction is ${ }^{19}$ 


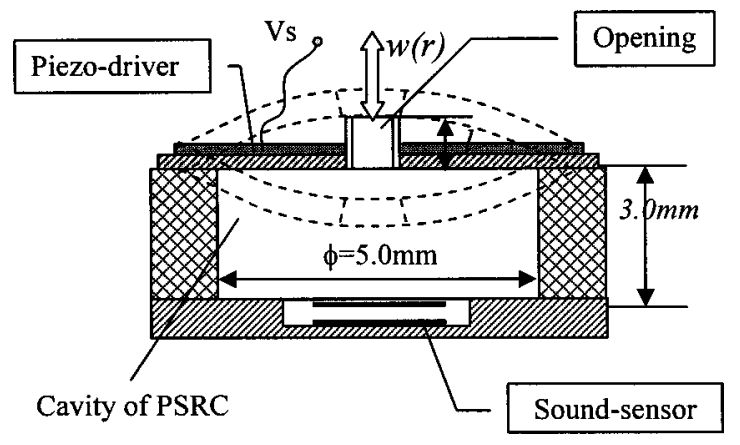

FIG. 1. Illustration of the concept of the PSRC sensor. One disk-type piezoelectric thin layer and a capacitance type sound sensing element are placed at each end of a small cavity. The piezoelectric disk produces a bending motion under voltage, generating a resonance sound field in the cavity, and radiating it through a center-opening. The acoustic sensing element monitors the resonance acoustic field and its changes. When an object is inserted near the opening of the cavity, the acoustic intensity $\Delta I$ and resonance frequency $f_{0}$ are altered due to reflection. These changes are detected by the sensing element.

$$
\begin{aligned}
& w(r)=\frac{e a^{2} h_{p} V_{E}}{4(1+\nu) D_{\mathrm{comp}}}\left(1-\frac{r^{2}}{a^{2}}\right)=w_{0}\left(1-\frac{r^{2}}{a^{2}}\right), \\
& w_{0}=\frac{e a^{2} h_{p} V_{E}}{4(1+\nu) D_{\mathrm{comp}}} ;
\end{aligned}
$$

where $a$ and $h_{p}$ are the radii and thickness of the piezoelectric disk, $\nu$ is Poisson's ratio, and $D_{\text {comp }}$ and $w_{0}$ are the elastic stiffness and the center displacement of the piezoelectric/metal laminate disk, respectively. This displacement will cause a cavity volume change $\Delta V$, given as

$$
\Delta V=\int_{0}^{a} 2 \pi r \cdot w(r) \cdot d r=\frac{1}{2} w_{0} A,
$$

where $A=\pi a^{2}$ is the cross-section area of the cavity.

The volume change of Eq. (2) will result in a pressure change $p_{1}$ in the cavity. According to ideal gas, $P_{0} V_{0}^{\gamma}$ should be a constant. As a result, under small displacement conditions $\left(\Delta V \ll V_{0}\right)$, we have

$$
p_{1} \approx-\rho_{0} c_{0}^{2} \frac{A}{2 V_{0}} w_{0},
$$

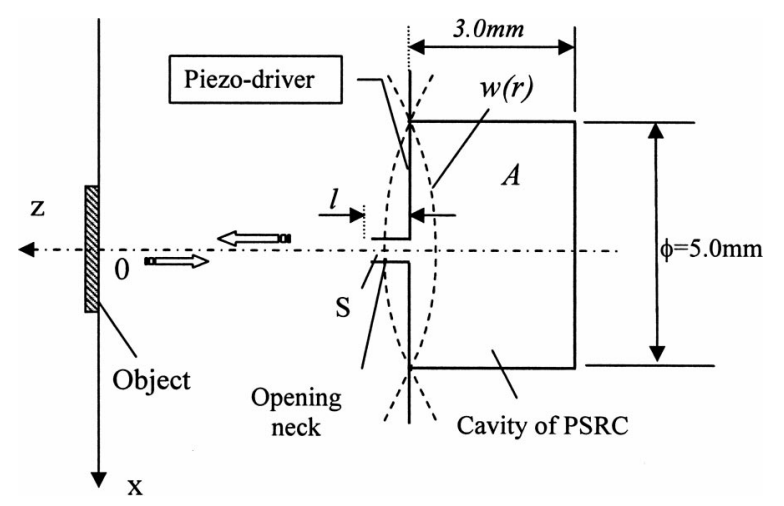

FIG. 2. Illustration of the operational principle of the PSRC position sensor. where $c_{0}$ and $\rho_{0}$ are the sound velocity and air density, respectively. The pressure change $p_{1}$ will exert an apparent force on the air column of the center-opening, given as

$$
F=p_{1} S=-\rho_{0} c_{0}^{2} \frac{S A}{2 V_{0}} w_{0},
$$

where $S$ is the cross-sectional area of the center-opening.

The cavity volume change $\Delta V$ caused by the piezoelectric disk is equal to that of the air column in the centeropening. Thus, we have $\Delta V=S \cdot \xi$ and $w_{0}=(2 S / A) \xi$, where $\xi$ is the air column displacement. Since $\xi$ may be much bigger than $w_{0}$, the cavity can act to amplify sound. A sound amplification function is often designated as a Helmholtz resonator. $^{20,21}$ Equation (4) can then be rewritten as

$$
\begin{aligned}
& F=-\rho_{0} c_{0}^{2} \frac{S^{2}}{V_{0}} \xi=-K_{M} \xi, \\
& K_{M}=\rho_{0} c_{0}^{2} \frac{S^{2}}{V_{0}},
\end{aligned}
$$

where $K_{M}$ is an apparent spring constant of the compressible media in the cavity, and the corresponding apparent spring compliance is $C_{M}=1 / K_{M}$. The air column in the centeropening thus acts like a "mass-spring" system, with mass $M_{M}$ and spring constant $K_{M}$. The motion equation of this forced mass-spring system under ac voltage $V_{E}$ is

$$
\left(M_{M}+M_{r}\right) \frac{d^{2} \xi}{d t^{2}}+\left(R_{M}+R_{r}\right) \frac{d \xi}{d t}+\frac{1}{C_{M}} \xi=F,
$$

where $R_{M}$ is the friction resistance between the air column and center-opening. Equation (6) describes a forced harmonic oscillation of the mass-spring system.

In applied acoustics, the volume-velocity $U[=(d \xi / d t) S]$ and the pressure variable $p$ are often used together as a lumped parameter. ${ }^{20}$ Because $F$ is sinusoidal (i.e., $F=S p_{A} e^{j \omega t}$ ), Eq. (6) can be rewritten as

$$
\left(M_{A}+M_{\mathrm{Ar}}\right) \frac{d U}{d t}+\left(R_{A}+R_{\mathrm{Ar}}\right) U+\frac{1}{C_{A}} \int U d t=p_{A} e^{j \omega t},
$$

where $M_{A}=M_{M} / S^{2}, R_{A}=R_{M} / S^{2}$ and $C_{A}=C_{M} S^{2}$ are the acoustic mass, acoustic resistance, and acoustic compliance of the air column in the center-opening, respectively; and $M_{\mathrm{Ar}}=M_{r} / S^{2}$ and $R_{\mathrm{Ar}}=R_{r} / S^{2}$ are the acoustic attachment mass and acoustic radiation resistance, respectively. Assuming harmonic motion, the solution to Eq. (7) is

$$
U=\frac{p_{1}}{Z_{A}}, \quad Z_{A}=\left(R_{A}+R_{\mathrm{Ar}}\right)+j\left[\omega\left(M_{A}+M_{\mathrm{Ar}}\right)-1 / \omega C_{A}\right]
$$

where $Z_{A}$ is the acoustic impedance of this vibration system. From Eq. (8), the volume-velocity $U$ of the air flowing through the center-opening is inversely proportion to $Z_{A}$. At the resonance state condition, $j\left[\omega\left(M_{A}+M_{\mathrm{Ar}}\right)-1 / \omega C_{A}\right]$ $=0$, and the pressure oscillations inside the cavity are relatively large; consequently, the volume-velocity $U$ in the center-opening is maximum. 


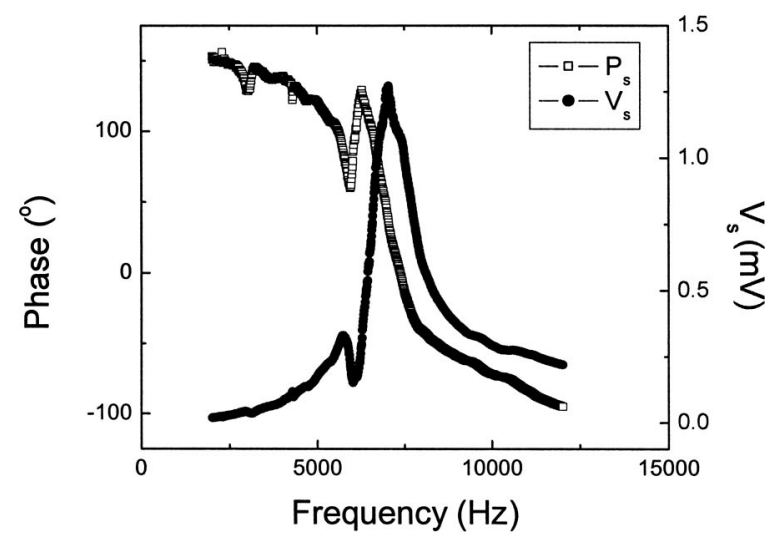

FIG. 3. Spectrum of cavity taken from the PSRC prototype using lock-in amplifier.

In the free condition, the resonance frequency is $\omega_{0}$ $=1 /\left(M_{A} C_{A}\right)^{1 / 2}$. However, the cavity produces a strong radiation and a resonance sound field; thus, the resonance frequency is shifted by $M_{\mathrm{Ar}}$ to $\omega_{1}=1 /\left[\left(M_{A}+M_{\mathrm{Ar}}\right) C_{A}\right]^{1 / 2}$. The change in acoustic impedance (both $R_{\mathrm{Ar}}$ and $M_{\mathrm{Ar}}$ ) caused by the sound radiation will result in a change in $U$. A large acoustic impedance will result in a small $U$; consequently, a small $V_{s}$ will be generated in the minisound sensor of the cavity. On the contrary, a small acoustic impedance will result in a large $U$; consequently, a large $V_{s}$ will be generated. If the working frequency is kept unchanged, the acoustic impedance change will result in a phase shift, rather than a resonance frequency change.

Insertion of an object into the resonance sound radiation field, near the center-opening of the cavity, will result in an acoustic impedance change due to reflection. Thus, by measurement of the sound signal change, we can detect an object's position. A relationship between the applied voltage $V_{E}$ and $U$ can be obtained by use of Eqs. (1), (4) and (8), given as

$$
\begin{aligned}
U= & -\frac{\rho_{0} c_{0}^{2} A}{2 V_{0}} \frac{e a^{2} h_{p}}{4(1+\nu) D_{\mathrm{comp}}} \\
& \times \frac{V_{E}}{\left(R_{A}+R_{\mathrm{Ar}}\right)+j\left[\omega\left(M_{A}+M_{\mathrm{Ar}}\right)-\frac{1}{\omega C_{A}}\right]} .
\end{aligned}
$$

This is the primary equation which governs the operational principle of the PSRC position sensor. It provides a relationship between the volume velocity $U$, applied voltage, and acoustical impedance changes due to object position and displacement.

\section{PROTOTYPE CONSTRUCTION AND OPERATIONAL CONDITIONS}

A prototype PSRC is shown in Fig. 1. This prototype consists of one ferroelectric lead zirconate titanate thin layer with a diameter of $8 \mathrm{~mm}$ and a thickness of $100 \mu \mathrm{m}$, a ceramic cylindrical cavity of $5 \mathrm{~mm}$ in diameter and $3 \mathrm{~mm}$ in height, and a capacitance type minisound sensor. The PSRC prototype can generate a resonance acoustic field along its axial direction. Figure 3 shows the acoustical signal

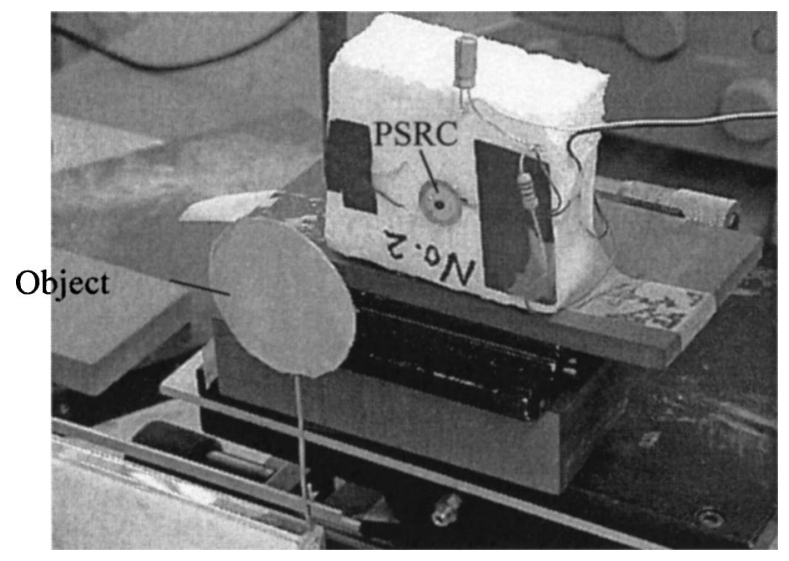

FIG. 4. Experimental setup for object position detection.

response-frequency spectrum.

A convenient method to operate a PSRC sensor is under constant frequency conditions. When an object is inserted into the acoustic radiation field, an echo will be produced, causing an acoustic radiation impedance change. As a result, a signal shift (voltage and phase) can be detected using the mini-sound sensor. By monitoring both the phase and voltage changes of the resonance state of the PSRC, the object position (relative to the PSRC) can be accurately determined.

Experiments have revealed for best performance that the PSRC prototype should be operated in either $\pm 90^{\circ}$ phase states. There are several frequencies between 5 and $12 \mathrm{kHz}$, which have this phase condition. These phase states are illustrated in the spectrum given in Fig. 3. In the $\pm 90^{\circ}$ phase states, both the phase and voltage were found to be significantly more sensitive to small displacements of the object, than in a $0^{\circ}$ phase state. In addition, for $\pm 90^{\circ}$ phase states, the PSRC prototype was more sensitive to both axial and transverse object displacements. The position sensitivity was found to depend on the initial position of the object, relative to the center-opening of the PSRC.

The response of the PSRC sensor to a moving object was determined at constant temperature and humidity conditions. A small drive voltage of $0.1-1.0 V_{\text {rms }}$ was used to excite the piezoelectric/metal laminate disk placed at one end of the cavity. A lock-in amplifier was used to monitor the signal from the minisound sensor placed at the other end. Investigations have shown that object insertion and/or object motion in the sound radiation field results in both phase and voltage changes.

\section{MEASUREMENT SETUP AND PROCEDURE}

The response of the PSRC prototype to a step-wise object movement was measured using the experimental setup shown in Fig. 4. A brass disk with a diameter of $30.0 \mathrm{~mm}$ served as the object, as shown in the figure. This object was translated along the $\hat{x}$ axis, which was vertical to the $\hat{z}$ axis of the PSRC prototype. The prototype was mounted on a linear slider, which could be translated along the $\hat{z}$ axis with precision.

The response of the PSRC sensor was measured using a lock-in amplifier method. A small ac voltage signal (5.92 or 
(a) Step displacement: 40 micrometer

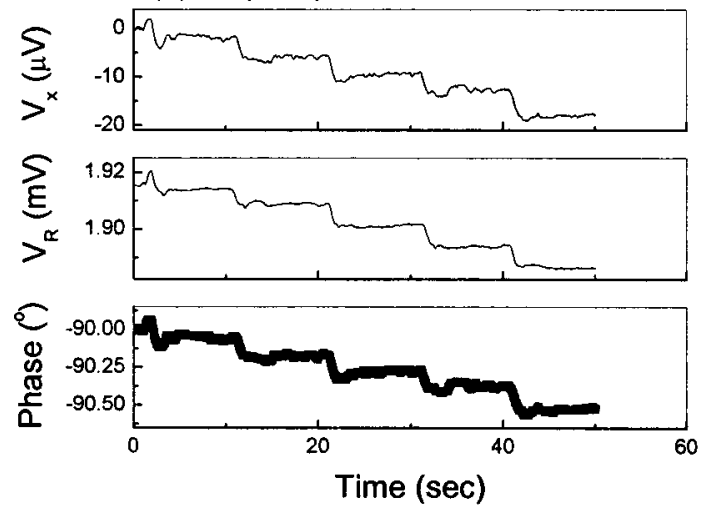

(c) Step displacement: 0 - 20 micrometer
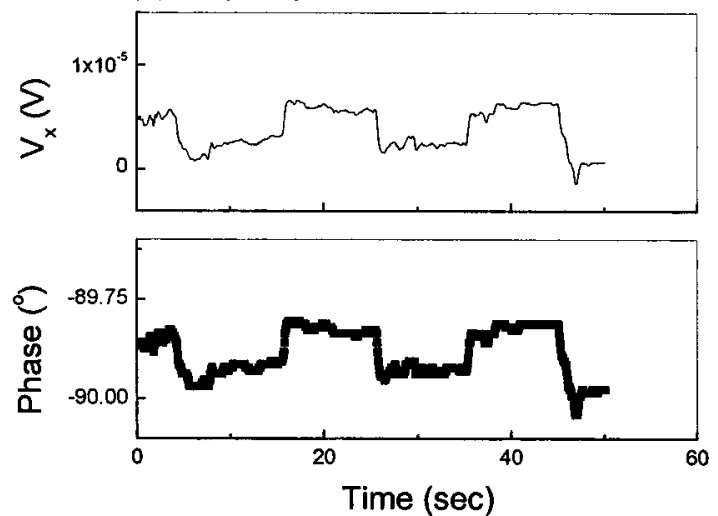

(b) Step displacement: -40 micrometer

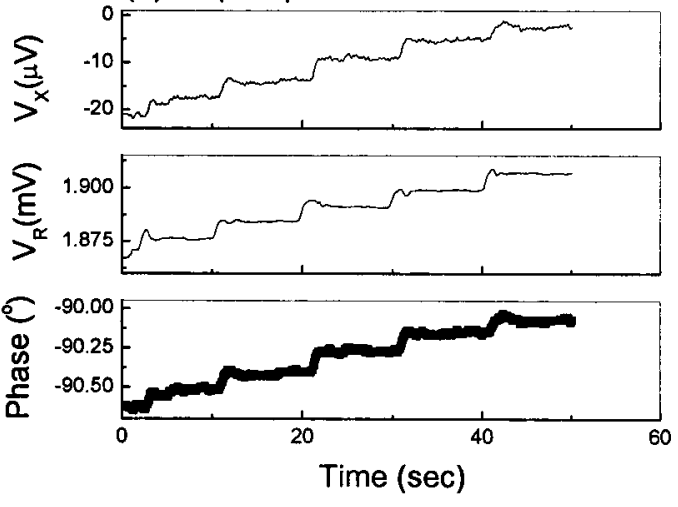

(d) Step displacement: 0-10 micrometer

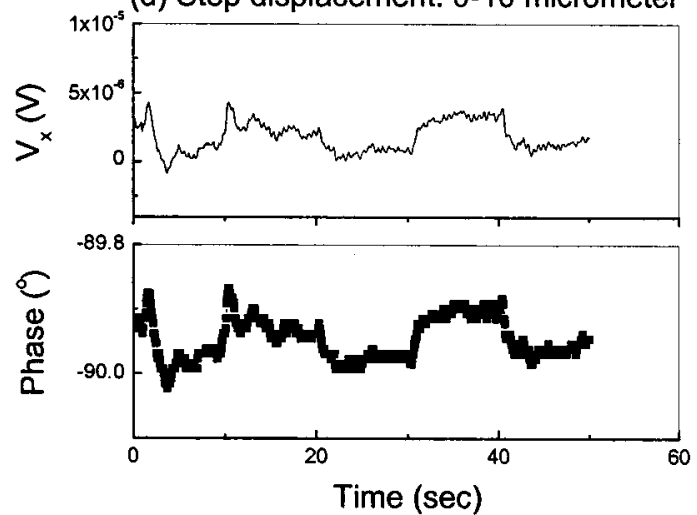

FIG. 5. Axial (longitudinal) position detection measurement (phase and voltage signal in PSRC) as a function of time for various step displacements of (a) 40, (b) -40 , (c) 20, and (d) $10 \mu \mathrm{m}$. The operational voltage and frequency were $0.9 V_{\text {rms }}$ and $11.699 \mathrm{kHz}\left(-90^{\circ}\right.$ phase state), respectively.

$11.70 \mathrm{kHz})$ of $0.1-0.9 \quad V_{\mathrm{rms}}$ was used to drive the piezoelectric/metal laminate displaced at one end of the cavity. A minisound sensor was placed at the other end, whose output signal was amplified. The detection signals (both the voltage and phase) were scanned and recorded by the lock-in amplifier, monitoring phase and voltage changes. The drive voltage was kept constant; thus measured changes can be attributed to object position changes. Because the drive voltage and input current were small, the power consumption was $<1 \mathrm{~mW}$.

\section{CHARACTERIZATION OF PSRC PROTOTYPE PERFORMANCE AS POSITION SENSOR}

A series of characterization experiments were performed to determine the PSRC prototype performance as a position sensor. These included determination of (i) the longitudinal sensitivity to object position along the $\hat{z}$ axis; (ii) the transverse sensitivity to object position, in directions vertical to the acoustic radiation field; and (iii) the range over which an object's position can be quantitatively detected.

\section{A. Axial displacement}

In order to determine the position resolution along the acoustic radiation direction, the object was fixed at a position of $15 \mathrm{~mm}$ in front of the center-opening of the PSRC. The PSRC was operated at a frequency of $11.7 \mathrm{kHz}\left(-90^{\circ}\right.$ phase state), using a small ac drive voltage of $0.9 V_{\text {rms }}$. The PSRC was then translated along the $\hat{z}$ axis at displacement steps of $\pm 40,20$, and $10 \mu \mathrm{m}$. In order to determine the position resolution of the PSRC prototype, the voltage and phase were continuously measured during translation.

Figures 5(a)-5(d) show the output voltage and phase for the PSRC prototype as a function of position for various step displacements of $40,-40,20$, and $10 \mu \mathrm{m}$, respectively. The time interval between step displacements was held constant at $10 \mathrm{~s}$ for all measurements. In Figs. 5(a) and 5(b), it can be seen that the PSRC prototype produces repeatable voltage and phase changes, in response to an object translation of either $\pm 40 \mu \mathrm{m}$. For smaller translations, the voltage and phase were both found to be linear functions of the object position. Figures 5(c) and 5(d) show the voltage and phase as a function of position using smaller translation steps of 20 and $10 \mu \mathrm{m}$, respectively. In these figures, it can be seen that the minimum detectable displacement resolution is $<10 \mu \mathrm{m}$. For example, a $20 \mu \mathrm{m}$ position change resulted in a phase change $(\Delta \theta)$ of $\sim 0.12^{\circ}$ and a voltage change $\left(\Delta V_{x}\right)$ of $\sim 5$ $\mu \mathrm{V}$; and a $10 \mu \mathrm{m}$ position change resulted in $\Delta \theta \sim 0.06^{\circ}$ and $\Delta V_{x} \sim 2.5 \mu \mathrm{V}$. The rates of phase and signal change to axial position translation along $\hat{z}$ were $6 \times 10^{-3} \% / \mu \mathrm{m}$ and 0.25 $\mu \mathrm{V} / \mu \mathrm{m}$, respectively.

Larger-range axial displacements $(10<z<200 \mathrm{~mm})$ were also measured, in order to fully characterize the position sensor's resolution capabilities of object detection. Figure 6 shows the phase and voltage as a function of the ob- 


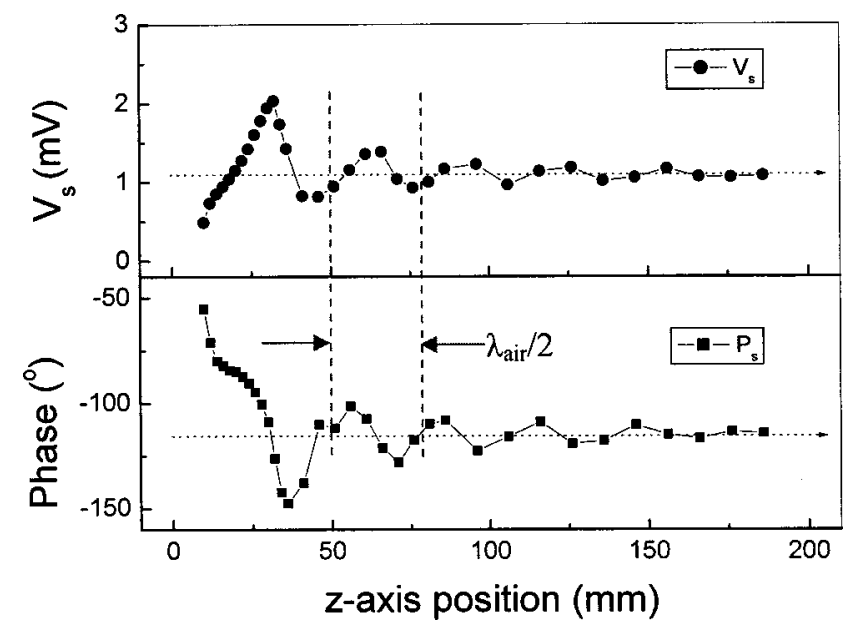

FIG. 6. Illustration of capabilities of PSRC sensor for large range axial (longitudinal) position detection. Phase and voltage as a function of the $\hat{z}$-axis position. The operational voltage and frequency were $0.5 V_{\text {rms }}$ and $5.92 \mathrm{kHz}$, respectively.

ject's $\hat{z}$-axis position. The operational voltage and frequency were $0.5 V_{\text {rms }}$ and $5.92 \mathrm{kHz}$, respectively. It can be seen that $V_{s}$ and $\theta$ are exponentially attenuated oscillation functions of the object's $\hat{z}$-axis position. The wave length of the exponentially attenuated oscillation (wave) is about half $(\sim 30 \mathrm{~mm})$ of that of the sound wave $(f=5.9 \mathrm{kHz})$. Moreover, a $90^{\circ}$ phase difference between $V_{s}$ and $\theta$ was observed. These results show for some special position that a standing wave (between the object and PSRC cavity bottom) is established, due to interference. The exponential attenuation contains information that is a signature of the object's large axial range position change.

\section{B. Transverse displacement}

Experimental measurements revealed that the PSRC is also sensitive to the transverse position and/or displacement of an object. In order to determine the transverse resolution along the $\hat{x}$ direction, which is vertical to the acoustic radiation field, the object was placed at different positions in front of the center-opening of the PSRC and step-wise translated from one side to another. The PSRC operational frequency was $5.92 \mathrm{kHz}\left(+90^{\circ}\right.$ phase state), and the drive voltage was $0.1 V_{\text {rms }}$. The object was step-wise translated by $5 \mathrm{~mm}$ every $30 \mathrm{~s}$ from right towards left.

Figures 7(a)-7(c) show the sensor output voltage as a function of the transverse position at various positions of $z$ $=1,5$, and $10 \mathrm{~mm}$, respectively. It can be seen that the phase is dependent not only on the axial position of an object, but also on transverse object displacements over a distance of $\sim 100 \mathrm{~mm}$. A sensitive area of $\sim 60 \mathrm{~mm}$ (or $2 \times$ the object's diameter) was found between $-22<\times<38 \mathrm{~mm}$, where $\Delta \theta$ was a sinusoidal-like function of position. The maximum phase change caused by object displacement (from center to side positions) was $\sim 36^{\circ}$ for $z=10 \mathrm{~mm}$ and $57^{\circ}$ for $z$ $=5 \mathrm{~mm}$. The average value of $\delta \theta / \delta x$ was $1.2 \times 10^{-3} \% / \mu \mathrm{m}$ for $z=10 \mathrm{~mm}$ and $1.9 \times 10^{-3} \% / \mu \mathrm{m}$ for $z=5 \mathrm{~mm}$.

When the object was placed close to the PSRC $(z$ $\leqslant 1 \mathrm{~mm}$ ), the phase changed from $\sim 90^{\circ}$ to $\sim 180^{\circ}$, as the

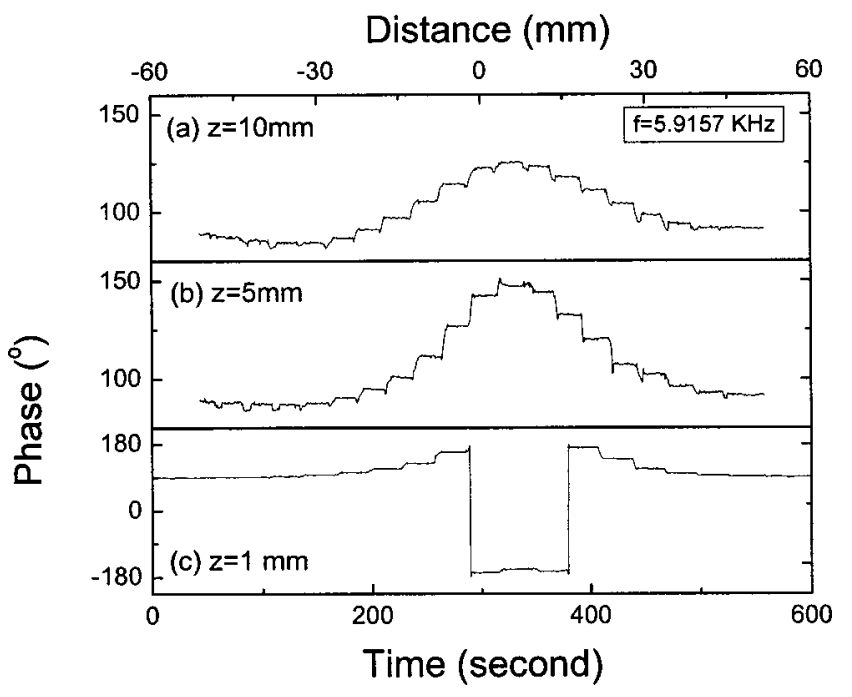

FIG. 7. Transverse position detection measurement. Phase signal in PSRC as a function of $x$-axis position and time for (a) $z=10 \mathrm{~mm}$, (b) $z=5 \mathrm{~mm}$, and (c) $z=1 \mathrm{~mm}$. The object was translated along the $x$ axis in a step-wise manner at $5 \mathrm{~mm}$ every $30 \mathrm{~s}$. The operational voltage and frequency were $0.1 V_{\mathrm{rms}}$ and $5.92 \mathrm{kHz}\left(90^{\circ}\right.$ phase state), respectively.

object was translated from side to center. Near the center location, the phase rapidly dropped to $-180^{\circ}$ and remained constant in the vicinity of the center-opening $(2 \times x$ $<14 \mathrm{~mm}$ ). These results demonstrate that a standing wave is formed when the object is close to the center-opening. Near this position, reflection is so strong that the echo and incident radiation waves interfere with each other, resulting in a standing wave between the object and PSRC cavity bottom.

Transverse displacements also resulted in a voltage change, which were shifted by $90^{\circ}$ with respect to the phase. In the vicinity of the center-opening, the phase is maximum, however the voltage is minimum, as shown in Fig. 8. Also, the relative phase change was much larger than the voltage change. This is because the change in acoustic radiation impedance caused by object displacement is dominately a reactance.

The results of this section demonstrate that our prototype

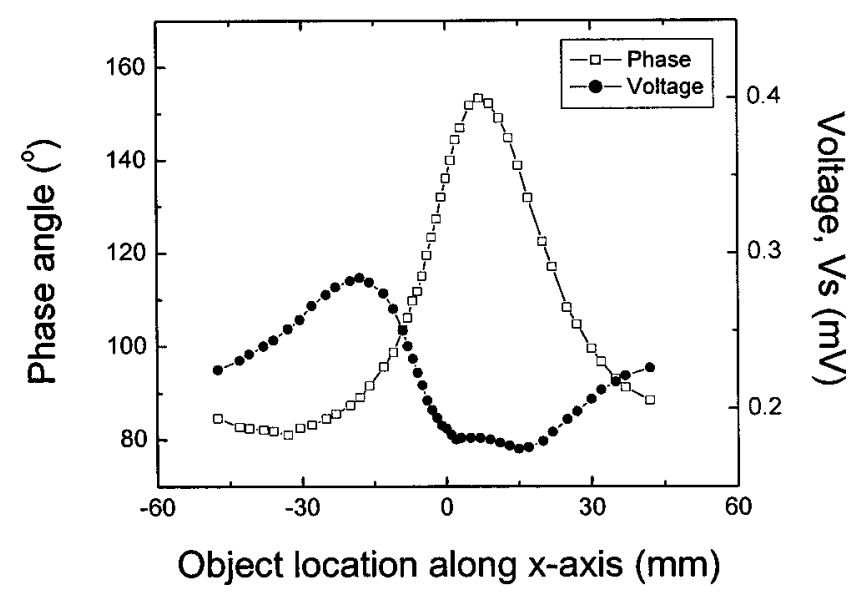

FIG. 8. Phase and voltage as a function of $x$-axis position for $z=10 \mathrm{~mm}$. The operational voltage and frequency were $0.1 V_{\mathrm{rms}}$ and $5.916 \mathrm{kHz}\left(90^{\circ}\right.$ phase state), respectively. 
PSRC sensor has good sensitivity to small transverse displacements. The transverse position resolution is $<100 \mu \mathrm{m}$. Over larger position ranges, the signal outputs (phase and voltage) are sinusoidal-like functions of position; but over small ranges they are linearly proportional to the object position.

Our resonance acoustic field position sensor is significantly different from conventional time-of-flight method, in which an ultrasonic transducer sends a very sharp pulse towards an object and measures the echo return time, or cwbased time-delay estimation method. Our PSRC is operated in a low-frequency $(5-12 \mathrm{kHz})$ harmonic resonance acoustic field. In addition, the PSRC prototype offers the potential of accurate noncontact object detection in either gaseous or fluidic compressible media.

\section{DISCUSSION}

A concept for object position detection has been developed. It is the PSRC, which uses the change in resonance sound field as a sensing mechanism. It is a simple, compact, robust, and light weight sensor. It is operated in lowfrequency resonance state under a very low working voltages $(<1 \mathrm{~V})$ and with extremely low power consumptions $(<1$ $\mathrm{mW}$ ). Investigations of a PSRC prototype have demonstrated a position sensitivity limit of $<10 \mu \mathrm{m}$, and a linear signal (phase and voltage) with object (small) position change. It offers a simple method to detect inserted objects and their motion in a compressible media, such as air or water, with high precision.

\section{ACKNOWLEDGMENTS}

The authors gratefully acknowledge the support of the Office of Naval Research under Grant Nos. N000140210340, N000140210126, and MURI N000140110761.

${ }^{1}$ V. Magori, 1994 IEEE Ultrasonic Symposium, 1994, p. 472.

${ }^{2}$ F. Ichikawa and T. Ohki, J. Acoust. Soc. Am. 90, 3384 (1991).

${ }^{3}$ G. Bucci and C. Landi, IEEE Trans. Instrum. Meas. 46, 1241 (1997).

${ }^{4}$ K. C. Eder and R. V. Krzyanowski, J. Acoust. Soc. Am. 83, 1710 (1988),

${ }^{5}$ C. M. Koukovinis, J. Acoust. Soc. Am. 80, 1277 (1986)

${ }^{6}$ R. M. White and S. Wenzel, J. Acoust. Soc. Am. 99, 2639 (1996).

${ }^{7}$ J. Yang, W.-S. Gan, and S.-E. Tan, Acoust. Resea. Lett. Online 4, 47 (2003).

${ }^{8}$ D. A. Stubbs and R. E. Dutton, JOM 48, 29 (1996).

${ }^{9}$ A. Warszawski, Y. Rosenfeld, and I. Shohet, J. Comput. Civ. Eng. 10, 67 (1996).

${ }^{10}$ K. Balasubramaniam et al., Rev. Sci. Instrum. 70, 4618 (1999).

${ }^{11}$ H. Peremans, J. Acoust. Soc. Am. 102, 1567 (1997).

${ }^{12}$ T. S. Jang, S. S. Lee, I. B. Kwon, W. J. Lee, and J. J. Lee, IEEE Trans. Ultrason. Ferroelectr. Freq. Control 49, 776 (2002).

${ }^{13}$ S. Shinohara, H. Yoshida, H. Ikeda, K.-I. Nishide, and M. Sumi, IEEE Trans. Instrum. Meas. 41, 40 (1992).

${ }^{14}$ N. E. Pears, IEEE Proceedings IROS, 1996, p. 81

${ }^{15}$ J. C. Piquette and A. E. Paolero, J. Acoust. Soc. Am. 113, 1518 (2003).

${ }^{16}$ X. Li, E. Lasson, M. Sheplak, and J. Li, IEEE J. Ocean. Eng. 27, 47 (2002).

${ }^{17}$ X. Li, R. Wu, M. Sheplak, and J. Li, IEE Proc. F, Radar, Sonar, Naviga. 149, 53 (2002).

${ }^{18}$ C.-C. F. Tong and E. J. F. Barbieri, IEEE Trans. Instrum. Meas. 50, 1324 (2001).

${ }^{19}$ S. Dong, K. Uchino, L. Li, and D. Viehland, J. Intell. Mater. Syst. Struct. (submitted).

${ }^{20}$ A. D. Pierce, Acoustics (Acoust. Soc. Am, Woodbury, NY, 1989).

${ }^{21}$ G. Du, Z. Zhu, and X. Gong, Basic Acoustics (Shengxue Jichu) (Shanghai Science and Technology, Shanghai, China, 1981) (in Chinese). 